Check for updates

Cite this: Phys. Chem. Chem. Phys., 2020, 22, 1983

Received 18th October 2019, Accepted 11th December 2019

DOI: $10.1039 / c 9 c p 05682 c$

rsc.li/pccp

\section{Simulation of Raman and Raman optical activity of saccharides in solution $\dagger$}

\author{
Vladimír Palivec, (D) $\ddagger^{a}$ Vladimír Kopecký Jr., (DD ${ }^{b}$ Pavel Jungwirth, (D) ${ }^{a}$ Petr Bouř, (D) ${ }^{a}$ \\ Jakub Kaminský (D) *ac and Hector Martinez-Seara (D) *a
}

\begin{abstract}
Structural studies of sugars in solution are challenging for most of the traditional analytical techniques. Raman and Raman optical activity (ROA) spectroscopies were found to be extremely convenient for this purpose. However, Raman and ROA spectra of saccharides are challenging to interpret and model due to saccharides' flexibility and polarity. In this study, we present an optimized computational protocol that enables the simulation of the spectra efficiently. Our protocol, which results in good agreement with experiments, combines molecular dynamics and density functional theory calculations. It further uses a smart optimization procedure and a novel adaptable scaling function. The numerical stability and accuracy of individual computational steps are evaluated by comparing simulated and experimental spectra of D-glucose, $D$-glucuronic acid, $N$-acetyl-D-glucosamine, methyl $\beta$-D-glucopyranoside, methyl $\beta$-D-glucuronide, and methyl $\beta$ - $N$-acetyl-D-glucosaminide. Overall, our Raman and ROA simulation protocol allows one to routinely and reliably calculate the spectra of small saccharides and opens the door to advanced applications, such as complete 3-dimensional structural determination by direct interpretation of the experimental spectra.
\end{abstract}

\section{Introduction}

Carbohydrates play a pivotal role in numerous functions in living organisms. They provide mechanical support such as in the case of cellulose. They form materials for energy storage, such as starch or glycogen. Alternatively, sugars such as ribose and deoxyribose are part of the genetic material. Other sugars play a significant role in the structure and function of the glycocalyx, ${ }^{1,2}$ which is a $10-50 \mathrm{~nm}$ thick layer found on the extracellular side of the plasma membrane of several eukaryotic cells. This layer and its components rich in sugars make possible cell-cell recognition and filtration of the surrounding biomolecules, and maintain the cell integrity. ${ }^{2}$ Its main components are proteins and their glycosylated variants, such as proteoglycans, and glycosaminoglycans. ${ }^{1}$

Better understanding of the function of saccharides requires a method for the determination of their flexible structures in solution. However, for sugars, such a task is particularly

\footnotetext{
${ }^{a}$ Institute of Organic Chemistry and Biochemistry, Czech Academy of Sciences, Flemingovo námèstí 542/2, CZ166 10, Prague 6, Czech Republic. E-mail: jakub.kaminsky@uochb.cas.cz, hseara@gmail.com

${ }^{b}$ Institute of Physics, Faculty of Mathematics and Physics, Charles University, Ke Karlovu 5, CZ121 16, Prague 2, Czech Republic

${ }^{c}$ Gilead Sciences \& IOCB Research Center, Czech Republic

$\dagger$ Electronic supplementary information (ESI) available. See DOI: 10.1039/c9cp05682c $\$$ PhD Student of University of Chemistry and Technology, Prague, Czech Republic.
}

difficult. Saccharide molecules, especially when functionalized (e.g., glycans), are often hard to crystallize, and they provide complex NMR spectra with broad and overlapping bands. ${ }^{3}$ Saccharides also usually lack UV-vis absorbing chromophores. Therefore, they cannot be easily investigated by standard UV-vis absorption or electronic circular dichroism spectroscopies. ${ }^{4}$ There are recent advancements in vacuum-ultraviolet electronic circular dichroism spectroscopy, but the technique is not yet able to routinely analyze and assign structural features of saccharides. ${ }^{5,6}$ Therefore, vibrational spectroscopies, in particular Raman and Raman optical activity (ROA), have been suggested as optimal for saccharides. Both methods, especially the chiral technique ROA, are sensitive to the structure of saccharides in solution. The ROA technique records the small but significant differences in the scattering of right- and left-circularly polarized light, and thus it increases the information about the structure in the spectrum, compared to standard Raman scattering. ${ }^{7}$ This is because ROA bands can be both positive and negative, which helps in their assignment, and the spectra are not only more sensitive to absolute chirality, but also to minor structure variations. The $200-1800 \mathrm{~cm}^{-1}$ spectral region commonly accessible by Raman and ROA spectroscopy rich in structural information is significantly wider and easier to obtain compared to those accessible to other vibrational techniques such as infrared (IR) and vibrational circular dichroism (VCD) spectroscopies.

Previously, Raman and ROA spectroscopies have been used, for example, to study the presence of secondary and tertiary 
structures of glycosaminoglycans in solution such as hyaluronan ${ }^{8}$ and heparan sulfate. ${ }^{9,10}$ In principle, this can be done by simply locating spectral changes as a function of the length of several hyaluronan moieties. However, assigning atomistic features directly from the experimental spectra is difficult. To a good approximation, measured Raman and ROA spectral shapes can be considered as weighted sums of the individual spectra of all molecular conformations adopted by the sugar. For this reason, previous Raman and ROA studies pointed out that the power of these spectroscopic techniques is significantly enhanced when coupled with computer simulations. ${ }^{11-14}$ Simulations not only provide the Raman and ROA spectra of a particular configuration but also allow one to predict the spectra of the real conformer mixture.

Early computational studies were significantly hampered by the excessive computer time needed for spectral simulations and interpretation. ${ }^{15,16}$ Nevertheless, it was quickly understood that for saccharides, with many semi-free rotating hydroxyl groups, single conformer models (especially if considered only in vacuum) cannot fully reproduce experimental data. Also, continuum (dielectric) solvent models provided only limited accuracy. ${ }^{13}$ So far, the best practical way of simulating the spectrum of a particular sugar moiety requires a combination of molecular dynamics and quantum-chemical calculations (typically density functional theory (DFT)), including at least some explicit solvent molecules in the quantum chemical computations. Such a procedure was used for example to study methyl $\beta$-D-glucopyranoside, ${ }^{13}$ where hundreds of explicit water molecules were employed within the monosaccharide solvation model. The same approach was recently used to study mannobiose disaccharides, where MD simulations, together with NMR spectroscopy, and DFT calculations were combined to produce Raman/ROA spectra. ${ }^{17}$ Another study focused on $\beta$-xylose showed that at least a $1 \mathrm{~nm}$ thick layer of water molecules is needed to model correctly the bulk aqueous environment. ${ }^{18}$ Other studies using explicit waters focused on subtle spectral modifications between D-glucose and D-galactose differing only in one chiral center ${ }^{19}$ and on a study of D-glucuronic acid and $N$-acetyl-D-glucosamine. ${ }^{20}$ One of the largest systems studied is the agarose hexamer. ${ }^{21}$ However, in the paper only continuum solvation without explicit water molecules is used, which was shown to be insufficient to properly capture Raman/ROA spectral features. $^{13}$

All these studies clearly hint at the necessity of including explicit waters when computing Raman and ROA spectra. Despite these important advancements, these studies neglected the anomeric equilibrium in sugars. This reason alone can explain several inconsistencies in their results like limited agreement of the calculated spectra with experiment. Only a few other studies, however, correctly accounted for the anomeric equilibrium when using explicit waters. One example is the study of glucose/mannose mixtures, ${ }^{12}$ where a hybrid solvation approach with the first solvation shell described quantum mechanically, together with the polarizable continuum model accounting for long range solvation effects, was used, resulting in reasonable agreement with experiments.
As of today, several protocols to model Raman and ROA spectra have been proposed with different degrees of success. ${ }^{12,13}$ In the present study, we compare various approaches to model Raman and ROA spectra of reducing monosaccharides and their methyl glycosides (methyl $\beta$-D-glucopyranoside [m $\beta$-glc], methyl $\beta$-D-glucuronide [m $\beta$-glcA], and methyl $\beta$ - $N$-acetyl-glucosaminide [m $\beta$-glcNAc], D-glucose [glc], D-glucuronic acid [glcA], and $N$-acetylD-glucosamine [glcNAc]) and compare them to original experimental data. As a result of our comparison, we propose a quantitative and computationally efficient protocol. This protocol builds on previous work, which we further optimize and improve in this current study. We also shed light on the need of taking into account the anomerization equilibrium when studying reducing sugars.

Finally, we consider the present study on monosaccharides as a first step allowing us to validate a universal methodology for computing Raman and ROA spectra, usable also for larger and more complicated systems in the future.

\section{Methods}

\subsection{Target monosaccharides}

In this work, in order to develop a Raman and ROA modeling protocol, we benchmarked several monosaccharides. Monosaccharides are the building blocks of more complex sugars, which our protocol also aims to cover in future studies. Here, we chose the two monosaccharides that comprise hyaluronan as the major glycocalyx component. These two sugars, $\mathrm{N}$-acetylD-glucosamine (glcNAc) and D-glucuronic acid (glcA), were complemented by D-glucose (glc), the structure of which is well known and, therefore, can be used to benchmark several aspects of the methodology. In aqueous solutions all three monosaccharides exist as a mixture of their $\alpha$ and $\beta$-anomeric forms. To have simpler reference systems devoid of the anomeric equilibrium, we also investigated non-reducing derivatives, i.e., methyl $\beta$-D-glucopyranoside ( $\mathrm{m} \beta$-glc), methyl $\beta$-D-glucuronide (m $\beta$-glcA), and methyl $\beta$ - $N$-acetyl-D-glucosaminide (m $\beta$-glcNAc). Only the pyranose forms of the sugars were considered, as significant occurrence of other forms is not likely in aqueous solutions. ${ }^{22}$ The structures of all studied molecules are depicted in Fig. 1.

\subsection{Experimental section}

All studied reducing monosaccharides glc, glcA, and glcNAc, as well as the methyl glycosides $\mathrm{m} \beta$-GlcA and $\mathrm{m} \beta$-GlcNAc, were purchased from Carbosynth. The concentration of sugars was 750-1000 mmol. Backscattered Raman and scattered circular polarization (SCP) ROA spectra were recorded on a ChiralRAMAN-2X (BioTools Inc.) spectrometer equipped with a $532 \mathrm{~nm}$ green laser. We first removed sample impurities causing unwanted fluorescence using active carbon. Then we quenched the residual fluorescence by leaving the sample in the laser beam for an hour before measurement. Spectra were accumulated in a fused silica cell $(3 \mathrm{~mm}$ optical path, $50 \mu \mathrm{L}$ sample volume) overnight $(\sim 12 \mathrm{~h})$. The laser power was $\approx 700 \mathrm{~mW}$. 

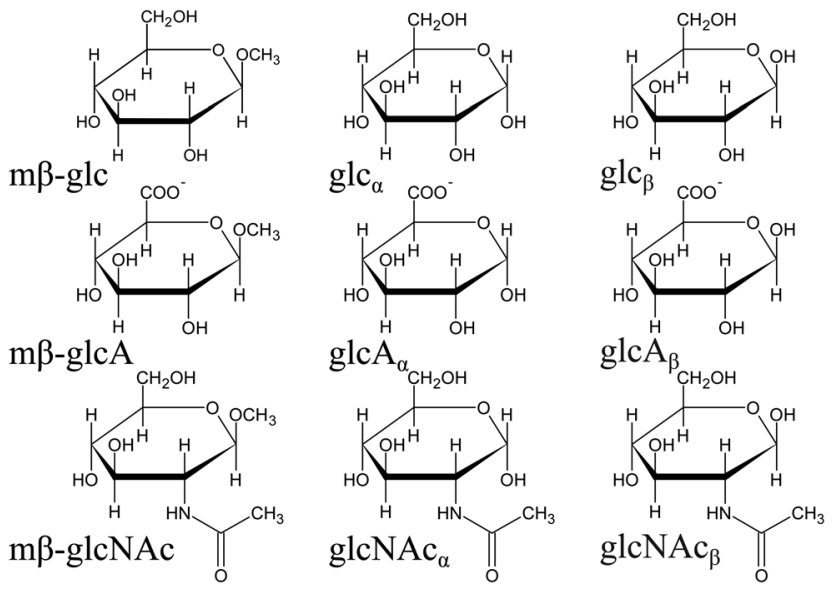

Fig. 1 The herein investigated monosaccharides.

The solvent signal was subtracted from the Raman spectra. Because this did not always eliminate broad background fluorescence from residual impurities, a minor polynomial baseline correction was done manually in some cases. The spectra were finally normalized to the accumulation time and we performed a relative intensity correction. ${ }^{23}$ Spectra of $\mathrm{m} \beta$-glc were taken from Cheeseman et al. ${ }^{13}$

\subsection{Simulation of Raman and ROA spectra}

Generation of the simulated spectra consisted of four stages: (1) generation of an ensemble of relevant structures including solvent positions using molecular dynamics (MD) simulations. (2) Calculation of the spectra using quantum chemical methods for the selected structures. (3) Minor adjustments of the calculated spectra, in particular scaling of calculated vibrational frequencies. (4) Weighting the spectra by conformer weights. A general scheme of the whole procedure is shown in Fig. 2.

2.3.1 Ensemble generation using molecular dynamics. An ensemble of structures for each monosaccharide in water was generated by performing molecular dynamics (MD) simulations using the program Gromacs-2016.4. ${ }^{24}$ For each sugar the simulated system contained a monosaccharide, initially in the ${ }^{4} C_{1}$ chair conformation, in a cubic box of $4 \times 4 \times 4 \mathrm{~nm}^{3}$ and $\sim 2150$ water molecules. For the D-glucuronic acid and its methyl derivative, we kept the system electroneutral by adding a single $\mathrm{Na}^{+}$ion. ${ }^{25}$ In MD simulations we used the glycam- $6 \mathrm{~h}^{26}$ and $\mathrm{OPC}^{27}$ force fields for the sugar and water, respectively. Long range electrostatic interactions were accounted for using $\mathrm{PME}^{28}$ with a $1 \mathrm{~nm}$ cut-off for the real part. Van der Waals interactions were treated with a cut-off of $1 \mathrm{~nm}$ with long range dispersion corrections for energy and pressure. ${ }^{29}$ Both Coulomb and van der Waals potentials were shifted to zero at the cut-off. Nearest neighbour searching was done with the Verlet list. All hydrogen containing bonds were constrained using the LINCS algorithm. ${ }^{30}$ Each system was first minimized using steepest descent minimization, followed by 100 ps heating up within an $N V T$ protocol to $310 \mathrm{~K}$, and finally equilibrated for 100 ps in the $N p T$ ensemble ( 1 bar). Afterwards, we ran a production $N p T$ simulation for $500 \mathrm{~ns}$ using a 2 fs time step. A Nosé-Hoover thermostat ${ }^{31,32}$ with a coupling constant of 1 ps controlled the temperature. A Parrinello-Rahman ${ }^{33}$ barostat using a time constant of 5 ps and compressibility of $4.5 \times$ $10^{-5}$ bar $^{-1}$ controlled the pressure. 100 uncorrelated snapshots, taken every $5 \mathrm{~ns}$, were saved for future analysis.

To simplify the subsequent $a b$ initio calculations with glcA, as a first approximation, we discarded around $7.5 \%$ of all gathered snapshots where the $\mathrm{Na}^{+}$ion distance to any atom of the monosaccharide was smaller than $5 \AA$.

2.3.2 Calculation of Raman and ROA spectra. The Raman and ROA frequencies and intensities were calculated using the Gaussian $16^{34}$ suite of programs. We used the B3LYP hybrid functional, in combination with the $6-311++G^{* *}$ basis set, as this combination provided in a similar context excellent results in terms of quality and computer efficiency previously. ${ }^{12}$ Various solvation schemes were tried, as summarized in Table 1. For every selected MD snapshot $(j)$, we took the monosaccharide coordinates with or without surrounding water molecules. Then, we optimized the geometry of the cropped system using various optimization schemes. The CPCM, MM, and hybrid simulation protocols employ ten optimization steps. In the case of the MM approach, Cartesian coordinates were used during the optimization as the internal coordinate optimization is $\sim 8$ times slower. The MM_w and hybrid_w optimization approaches freeze cropped water molecules and the sugar molecule is optimized until the gradient criteria are reached. With the hybrid_fo approach we optimize the whole system fully until the gradient criteria are reached. Finally, we have calculated the vibrational frequencies $\nu_{i j}^{*}$, and corresponding Raman, $I_{\mathrm{Raman}, i j}^{\mathrm{sim}}$, and ROA, $I_{\mathrm{ROA}, i j}^{\mathrm{sim}}$, intensities, where $i$ refers to a calculated vibrational mode of a snapshot $j$. Note that in this work we use interchangeably the terms vibrational frequencies $\nu_{i j}{ }^{*}$ and vibrational wavenumbers $\tilde{\nu}_{i j}{ }^{*}$, which are mutually connected as $\nu_{i j}{ }^{*}=c \cdot \tilde{\nu}_{i j}{ }^{*}$, where $c$ stands for the speed of light. We used the harmonic approximation and the far from resonance coupled-perturbed Kohn-Sham theory (CPKS) ${ }^{35,36}$ with the $532 \mathrm{~nm}$ excitation frequency corresponding to the green laser used in the experiment. In this work, the Raman and ROA spectra correspond to the scattered circular polarization (SCP) modulation scheme and backscattering $\left(180^{\circ}\right)$ detection.

All methods listed in Table 1 describe the monosaccharides at the DFT level of theory. When performing QM/MM calculations, we employ the ONIOM method ${ }^{37,38}$ with electrostatic embedding. For the force field description of the water and sugars, we used the TIP3P $\mathrm{P}^{39}$ and glycam- $6 \mathrm{~h}^{26}$ charges and Lennard-Jones parameters, respectively. For other force field parameters, we use the default values as in Gaussian16, however, note that their energetic contributions cancel in the ONIOM approach. Other variations of the simulation protocols are described in Tables S1-S3 in the ESI. $\dagger$ They address different optimization procedures, DFT functionals, basis sets, usage of QM water molecules, and convergence issues in the Raman and ROA spectra calculations.

2.3.3 Adjustments of the calculated spectra. The calculated vibrational frequencies, $\tilde{\nu}_{i j}{ }^{*}$, are usually slightly overestimated when using hybrid DFT approaches (see Section S4 in the ESI $\dagger$ ). ${ }^{35,40-45}$ Also, the use of a simple harmonic approximation can lead to further deviations of the frequencies. ${ }^{35,41}$ To account 


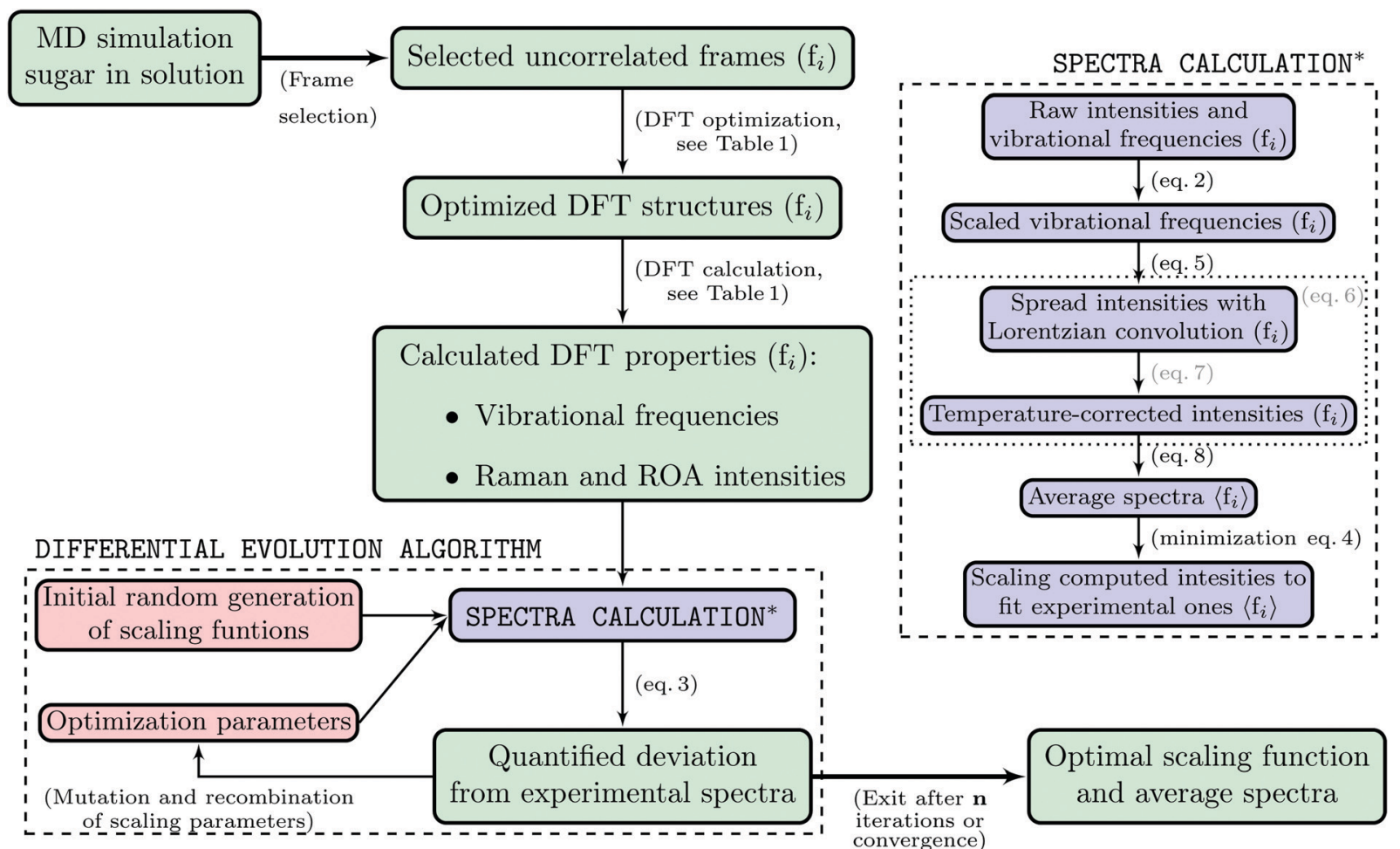

Fig. 2 Generation scheme of the simulated spectra, $I_{x}^{\operatorname{sim}}$.

Table 1 Principal solvation protocols used in this work

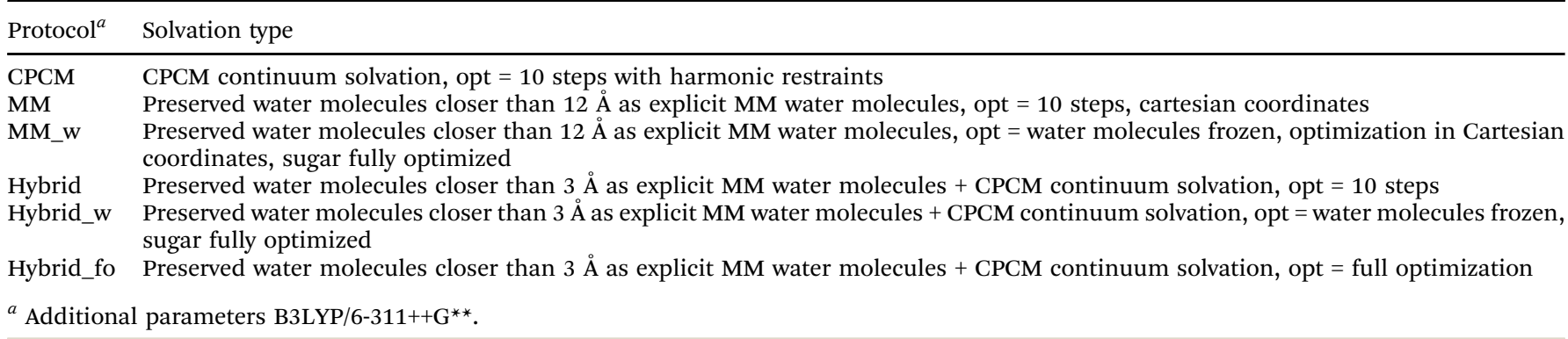

for these deviations, we adjusted ("scaled") the calculated vibrational frequencies. In this work, we used the following scaling function $\phi\left(\tilde{\nu}_{i j}^{*}\right)$.

$$
\phi\left(\tilde{\nu}_{i j}^{*}\right)=a \cdot \frac{1}{1+\mathrm{e}^{\frac{-\tilde{\nu}_{i j}^{*}+d}{c}}}+b \cdot\left(1-\frac{1}{1+\mathrm{e}^{\frac{-\tilde{\nu}_{i j}^{*}+d}{c}}}\right)
$$

The adjustable parameters $a, b, c$, and $d$ represent the high frequency region scaling factor $(a)$, the low frequency region scaling factor $(b)$, the frequency threshold between the low and high frequency regions (c), and a factor ensuring a smooth transition between these two regions $(d)$. Applying the function for each of the calculated vibrational frequencies, $\tilde{\nu}_{i j}{ }^{*}$, provides frequencies, $\tilde{\nu}_{i j}$, that are in better agreement with experiments.

$$
\tilde{\nu}_{i j}=\phi\left(\tilde{\nu}_{i j}^{*} ; a, b, c, d\right) \cdot \tilde{\nu}_{i j}^{*}
$$

Note that when $a=1$ and $b=1$, we obtain the original simulated spectra, $\tilde{\nu}_{i j}^{\text {sim }}$. The parameters $a, b, c$, and $d$ were optimized to fit best the experimental Raman and ROA spectra simultaneously by employing a cost function $\psi\left(a, b, c, d, \rho_{\text {Raman }}, \rho_{\text {ROA }}\right)$

$$
\psi=\chi_{\mathrm{Raman}}\left(a, b, c, d, \rho_{\mathrm{Raman}}\right) \cdot \chi_{\mathrm{ROA}}\left(a, b, c, d, \rho_{\mathrm{ROA}}\right) .
$$

Here

$$
\chi_{x}\left(a, b, c, d, \rho_{x}\right)=\sum_{\tilde{\nu}=\tilde{\nu}_{\min }}^{\tilde{\nu}_{\max }}\left|\rho_{x} \cdot I_{x}^{\operatorname{sim}}(a, b, c, d, \tilde{\nu})-I_{x}^{\exp }(\tilde{\nu})\right|,
$$

serves as an overall absolute difference between experimental $I_{x}^{\exp }$ and ensemble average calculated spectra $I_{x}^{\text {sim }}$, using the parameter $\rho_{x}$ to match the experimental and calculated intensities of a given spectrum. Note that all simulation results are shown with the optimized value of $\rho_{x}$. The average magnitudes 
of ROA and Raman intensities are experimentally related $\left(I_{\mathrm{ROA}} \approx I_{\text {Raman }} \cdot 10^{-4}\right)$, however, we optimize both spectral intensities separately. As a result, the ratio is not necessarily preserved during the optimization. More details about the proposed scaling function, i.e. how it works, what are its exact effects, and how it compares to non-scaled spectra/a simple scaling factor, can be found in Section S8 in the ESI. $\dagger$

In eqn (3), we use a product of errors, which worked well in our case, however, it was later pointed out that using a sum of errors avoids possible selective optimization of either the Raman or ROA spectrum. For the optimization procedure, we used a differential evolution algorithm using values of $0.7,[0.5,1]$, and 100 as crossover probability, differential weight, and population size parameters. Such optimization ultimately allows for a better match of the calculated intensities with experiments, providing most of the structural information. The presented Raman and ROA spectra correspond to the optimized ones using $a^{\text {optimal }}, b^{\text {optimal }}, c^{\text {optimal }}, d^{\text {optimal }}, \rho_{\mathrm{ROA}}^{\text {optimal }}$, and $\rho_{\text {Raman }}^{\text {optimal }}$.

2.3.4 Simulated spectral intensities. After scaling the frequencies, the simulated Raman and ROA spectra for a given snapshot $j$ are calculated as:

$$
I_{x, j}^{\operatorname{sim}}(\tilde{\nu})=T(\tilde{\nu}) \cdot \sum_{i=1}^{N_{\mathrm{vib}}} I_{x, i j}\left(\tilde{\nu}_{i j}\right) \cdot L\left(\tilde{\nu} ; \tilde{\nu}_{i j}\right),
$$

where

$$
L\left(\tilde{\nu} ; \tilde{\nu}_{i j}\right)=\frac{2}{\pi} \cdot \frac{\Gamma}{4\left(\tilde{\nu}-\tilde{\nu}_{i j}\right)^{2}+\Gamma^{2}}
$$

is a Lorentzian function using a full width at half maximum (FWHM) $\Gamma=7.5 \mathrm{~cm}^{-1}, N_{\text {vib }}$ is the number of calculated vibrational modes, and

$$
T(\tilde{\nu})=\frac{1}{\tilde{\nu}} \cdot \frac{1}{1-\mathrm{e}^{-\frac{\hbar \cdot \tilde{\nu}}{k_{\mathrm{B}} T}}}
$$

is a temperature correction factor, in our case applied at $310 \mathrm{~K}^{41}$ $x$ denotes either the Raman or ROA spectrum.

Raman, $I_{\mathrm{Raman}}^{\mathrm{sim}}(\tilde{\nu})$, and ROA, $I_{\mathrm{ROA}}^{\mathrm{sim}}(\tilde{\nu})$, spectra are obtained as an average over all snapshots, while the intensities are adjusted using the $\rho_{x}$ parameter

$$
I_{x}^{\operatorname{sim}}(\tilde{\nu})=\rho_{x} \cdot \frac{\sum_{j=1}^{N_{\text {sim }}} I_{x, j}^{\operatorname{sim}}(\tilde{\nu})}{N_{\text {sim }}},
$$

where $N_{\text {sim }}$ is the number of selected MD snapshots.

Final spectra were produced using 50000 optimization cycles using eqn (3) when the spectra did not change anymore.

2.3.5 Accounting for the $\alpha / \beta$ anomeric ratio. In the case of reducing monosaccharides (D-glucose, D-glucuronic acid, and $N$-acetyl-D-glucosamine) the $\alpha / \beta$ anomeric equilibrium has to be always considered. The calculated spectra, $I_{x}^{\operatorname{sim}, \alpha / \beta}$, were obtained as a weighted average of anomer spectra as follows

$$
I_{x}^{\mathrm{sim}, \alpha / \beta}=\left(1-x_{\beta}\right) I_{x}^{\mathrm{sim}, \alpha}+x_{\beta} I_{x}^{\mathrm{sim}, \beta},
$$

where $x$ is the fraction of the anomer, $I^{\mathrm{sim}, \alpha}$ is the average simulated spectrum of the anomer, and $I^{\operatorname{sim}, \beta}$ is the average simulated spectrum of the anomer. This equation was then used together with eqn (3) to optimize the scaling function parameters. In this work, we calculate the spectra of reducing sugars using experimental values of $x_{\beta}$ : D-glucose $-0.63,{ }^{22,46,47}$ D-glucuronic acid $-0.61,{ }^{48,49}$ and $N$-acetyl-D-glucosamine $-0.42 .^{50}$

\subsection{Assessment of the similarity of two spectra}

To facilitate the comparison of the tested methods (e.g., CPCM, MM, hybrid, hybrid_w, and hybrid_fo solvation models), we used an overlap integral $S$, which is defined as

$$
S=\frac{\int_{200}^{1800} I_{\mathrm{f}}(\tilde{\nu}) I_{\mathrm{g}}(\tilde{\nu}) \mathrm{d} \tilde{\nu}}{\sqrt{\int_{200}^{1800} I_{\mathrm{f}}(\tilde{\nu})^{2} \mathrm{~d} \tilde{\nu} \int_{200}^{1800} I_{\mathrm{g}}(\tilde{\nu})^{2} \mathrm{~d} \tilde{\nu}}}
$$

where $I_{\mathrm{f}}$ and $I_{\mathrm{g}}$ represent the two spectra that are to be compared. Upon comparing Raman spectra, the value of the overlap integral is in the range $(0,1)$, where values closer to 1 signal very similar spectra. Comparing ROA spectra yields a value in the range $(-1,1)$, where values close to 1 signal very similar spectra, while -1 would yield the exact mirror image. Note that in the case of methyl- $\beta$-glucopyranoside the integration limit was set to $(200,1490) \mathrm{cm}^{-1}$ because of insufficiently subtracted water signal appearing above that limit.

\section{Results}

\subsection{Comparison of simulated and experimental spectra}

Fig. 3 shows the experimental and simulated spectra of all studied monosaccharides. In the simulations, the hybrid solvation protocol from Table 1 was used. Overall, the computed spectra capture most of the experimental spectral features in all studied saccharides, that is relative Raman band intensities and for ROA also band signs, correctly. The low frequency region (200-800 $\left.\mathrm{cm}^{-1}\right)$ is reproduced with high accuracy with the exception of D-glucuronic acid, while for the high-frequency region (800-1800 $\mathrm{cm}^{-1}$ ), we observe larger deviations between calculated and experimental intensities, resulting in semiquantitative agreement only. The experimental peak in the case of $\mathrm{m} \beta$-glc close to $\sim 1640 \mathrm{~cm}^{-1}$ belongs to water bending and thus it was not reproduced by our calculations. Note that the spectra of glucose, glucuronic acid, and $N$-acetyl-D-glucosamine are composed of two independent spectra of $\alpha / \beta$ anomers. Incorporation of both anomers into the calculation is crucial and cannot be neglected as described in Section S3 in the ESI, $\dagger$ where the spectra of individual anomers can be found.

The spectra of methyl capped saccharides $(\mathrm{m} \beta$-glc, $\mathrm{m} \beta$-glcA and $m \beta$-glcNAc) are reproduced better than the spectra of nonmethylated sugars (glc, glcA, and glcNAc). Full optimization (hybrid_fo) can recover some of the originally non-reproduced peaks (Fig. S1-S3, S5, and S6 in the ESI $\dagger$ ), however, despite these minor improvements, the full optimization significantly worsened the results as a whole. This deterioration indicates that the variability of the ensemble obtained via MD is a crucial factor in the success of our protocol. Full minimization of the 

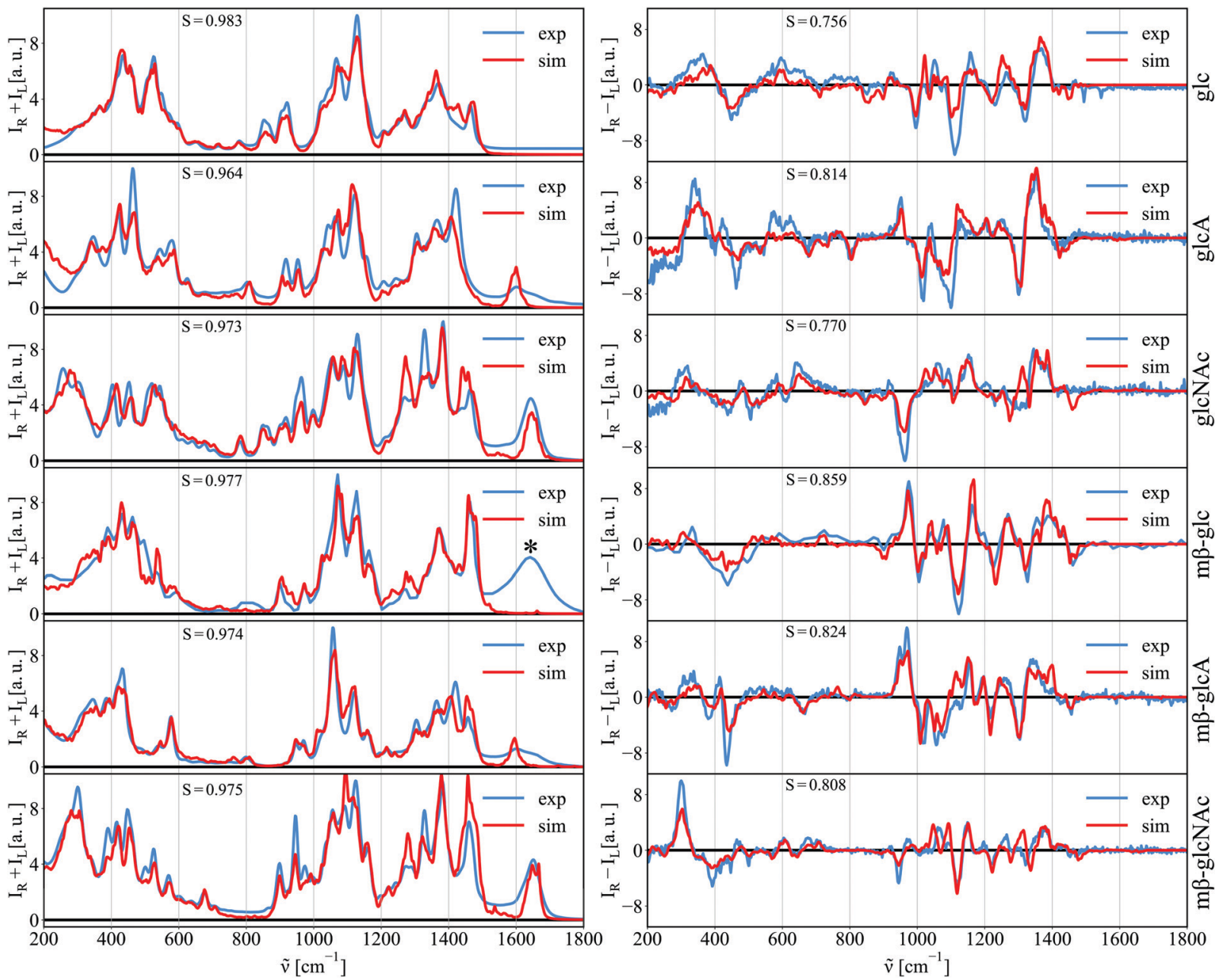

Fig. 3 Simulated and experimental Raman and ROA spectra of the investigated monosaccharides. The calculated spectra were obtained using the hybrid protocol (Table 1). Optimized frequency scaling functions together with the parameters are to be found in Fig. S1-S5 in the ESI. $\dagger{ }^{*} \mathrm{~m} \beta$-glc: leftover water band (not connected to the investigated saccharide).

extracted systems, which are just small regions of the complete phase space, reduces the number of local minima (configurations), impacting adversely the calculated spectra.

Overall, the hybrid computational protocol, which uses classically described water molecules, is fit to reproduce the main spectral features of the studied systems. Moreover, it provides similar quality to the results obtained when using QM described water molecules (for comparison, see Fig. S15 in the ESI $\dagger$ ). The hybrid protocol provides reasonably accurate results, with a much lower computational cost. Computational time was saved primarily by not using DFT for the water molecules. For a monosaccharide, e.g. glucose (24 atoms), the first coordination shell contains at least 15 water molecules. By avoiding the DFT treatment of this first coordination shell, we reduced the computational time $\sim 30$ times. Other significant savings originate from employing the partial optimization, being 3 to 8 times faster than full optimization. In this way, we achieved one of the main objectives of our study, making the computational protocol efficiently applicable also for bigger oligosaccharides.

\subsection{Estimate of experimental error}

As a simple approximate method of estimating the experimental error that can contribute to the disagreement between the experiment and theory, in Fig. 4 we compare the experimental ROA spectra of D-glucuronic acid and $N$-acetyl-D-glucosamine obtained by different experimental groups. Clearly, the reproducibility of the ROA intensities is limited. Moreover, in central regions, e.g., for glcA $1050-1150 \mathrm{~cm}^{-1}$, two experimental lines differ from each other more than they deviate from the calculation. Many factors contribute to such differences different experimental setups, subtraction of baselines, quality of the products, etc. Although this is not an ideal situation, we can use the error associated with the experimental spectra as an argument that our computed spectra achieve sufficient accuracy for interpreting experiments.

\subsection{Further optimization of the simulation protocol}

We devoted substantial attention to optimizing the spectra simulation protocol, that is to reduce the computational costs 


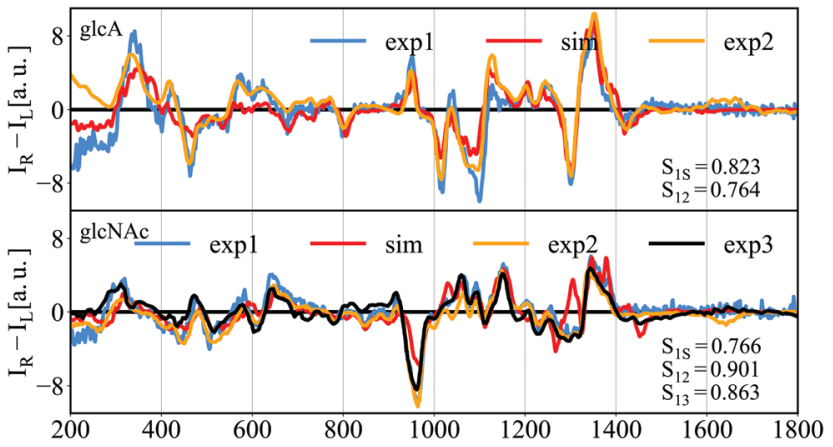

Fig. 4 Different experimental ROA spectra of D-glucuronic acid (glcA) and $\mathrm{N}$-acetyl-D-glucosamine (glcNAc) available. In orange spectra from ref. 20, in blue and black two independent spectra measured in this work (different spectrometer/sugar-provider). Simulation results obtained by the hybrid approach are in red.

while preserving reasonable agreement with the experimental data. We have considered multiple solvation methods, optimization procedures, functionals, and basis sets to calculate the Raman and ROA spectra.

Fig. 5 summarizes the performance of the CPCM, MM, MM_w, hybrid, hybrid_w, and hybrid_fo approaches in terms of overlap integral $S$. Proper treatment of the aqueous solvent surrounding the monosaccharide turned out to be a critical point. The relative performances of individual solvation models (Table 1) are shown in Fig. S1-S5 in the ESI. $\dagger$ We find that using only an implicit water solvent model can fail to reproduce experimental spectra, e.g., in the case of D-glucuronic acid (Fig. S2 in the ESI $\dagger$ ). Including several solvation layers of explicit water molecules (cutoff $12 \AA$, MM protocol ${ }^{18}$ ) results in a significant improvement over the CPCM approach. Full optimization of the sugar, while the water molecules are kept frozen (MM_w), yields even better results, but at increased computational cost. A combination of implicit and explicit water models (hybrid protocol) performs even better compared to the MM_w protocol with the advantage of treating explicitly a significantly smaller system and being faster. A modification of

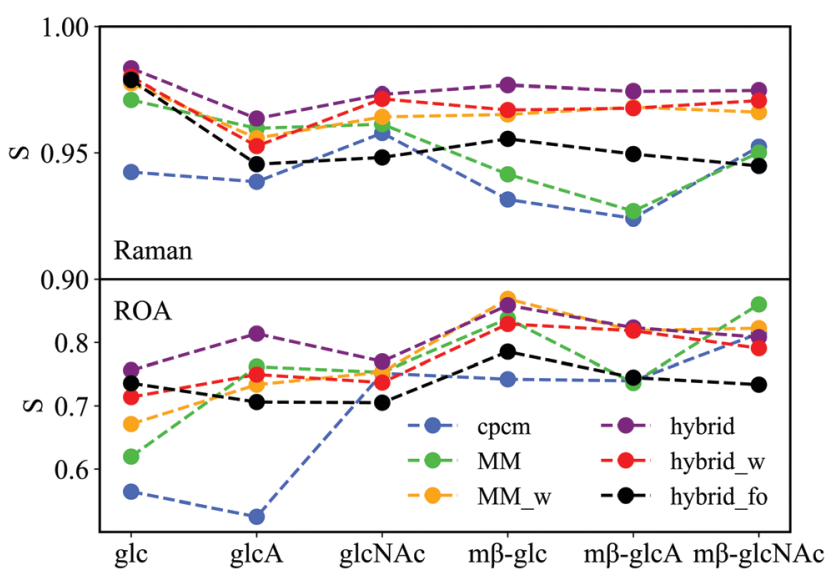

Fig. 5 Performance of the employed protocols (CPCM, MM, MM_W hybrid, hybrid_w, and hybrid_fo) in terms of the overlap integral S for the studied monosaccharide species. the hybrid protocol - hybrid_w - was found to behave similarly to MM_w, however, still worse than the hybrid. The full optimization (hybrid_fo) was found to poorly reproduce low frequency regions at a high computational cost. The performance in relative cpu times is: cpcm:mm:MM_w: hybrid: hybrid_w: hybrid_fo $=1: 3.7: 7.5: 1.7: 3: 14$. This results in average performance in terms of overlap integral for Raman: $0.941: 0.952: 0.966: 0.974: 0.968: 0.954$ and for ROA: 0.689 : $0.761: 0.778: 0.805: 0.773: 0.735$.

We conclude that the best performing approach is hybrid, also providing results at reasonable cost.

Next, we have tested the number of optimization steps prior to the Raman/ROA calculations. Fig. 6 shows the performance of the hybrid approach when using 0 (MD structures), 1, 3, 5, $10,30,50,100,200$, and 300 (full optimization) optimization steps. Both Raman and ROA spectra need 3-10 optimization steps, after which the spectra deteriorate and the error goes up. This is due to the bad description of low frequencies which becomes noticeable after 30 optimization steps. The optimal number of optimization steps is 10 , where the hybrid approach performs the best. Examples of spectra evolution as a function of the number of optimization steps of glucuronic acid and methyl $\beta$-D-glucuronide are shown in Fig. S9 and S10 in the ESI. $\dagger$ We have further tested 'Normal mode optimization', which is commonly used in the literature. ${ }^{51}$ It turned out to produce similar results to the hybrid approach with the exception of the ROA spectrum of methyl $\beta$-D-glucuronide, where the frequencies near $1000 \mathrm{~cm}^{-1}$ were poorly described (Fig. S8 in the ESI $\dagger$ ).

Next, we have tested 4 different DFT functionals (B3LYP, CAMB3LYP, M06L, and $\omega$ B97XD). All functionals resulted in qualitatively similar results, however, the B3LYP functional performed the best as shown in Fig. 7. As the B3LYP functional has been thoroughly tested for Raman and ROA spectra calculations, ${ }^{12,52-54}$ we have selected it for our optimized hybrid protocol.

The selection of a suitable basis set was also considered. Employing the hybrid solvation protocol we tested the cheaper

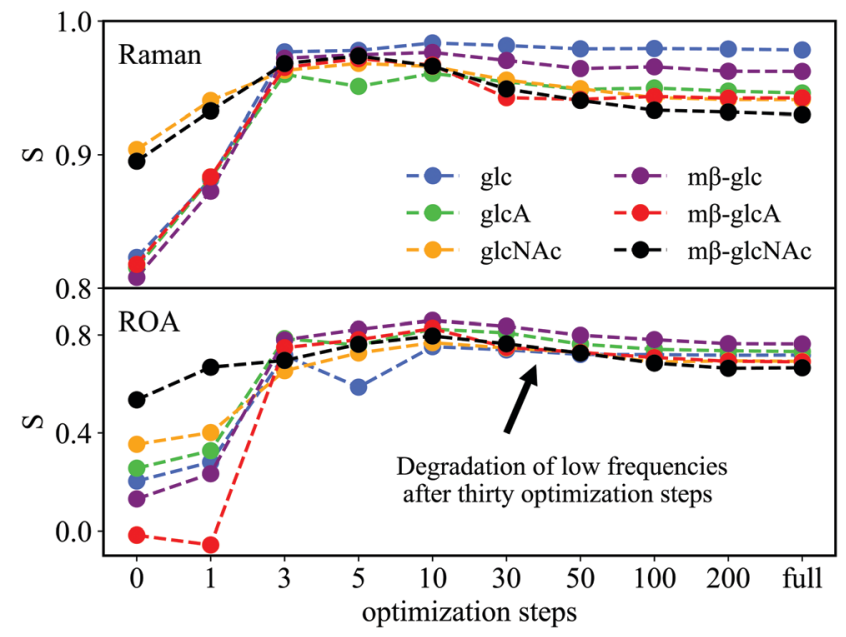

Fig. 6 Performance of the hybrid protocol when using different numbers of optimization steps prior to spectra calculations. 


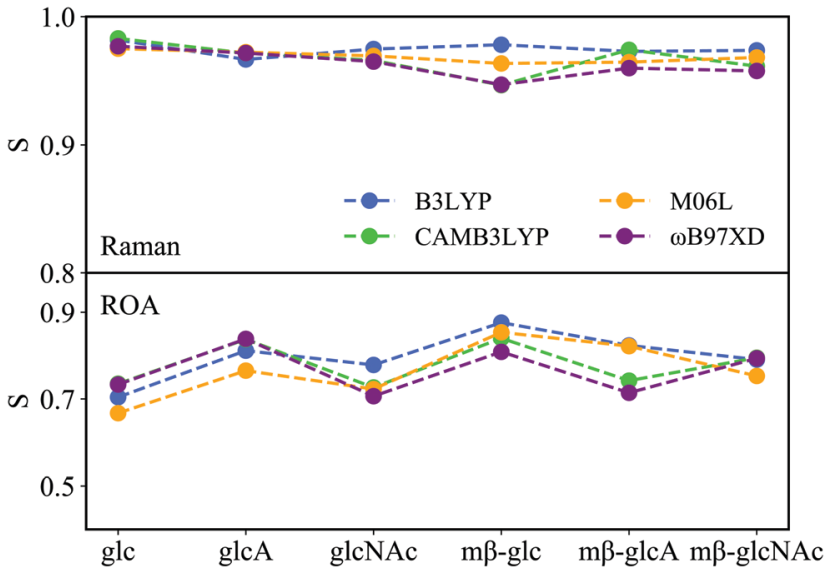

Fig. 7 Performance of the hybrid protocol when using different DFT functionals.

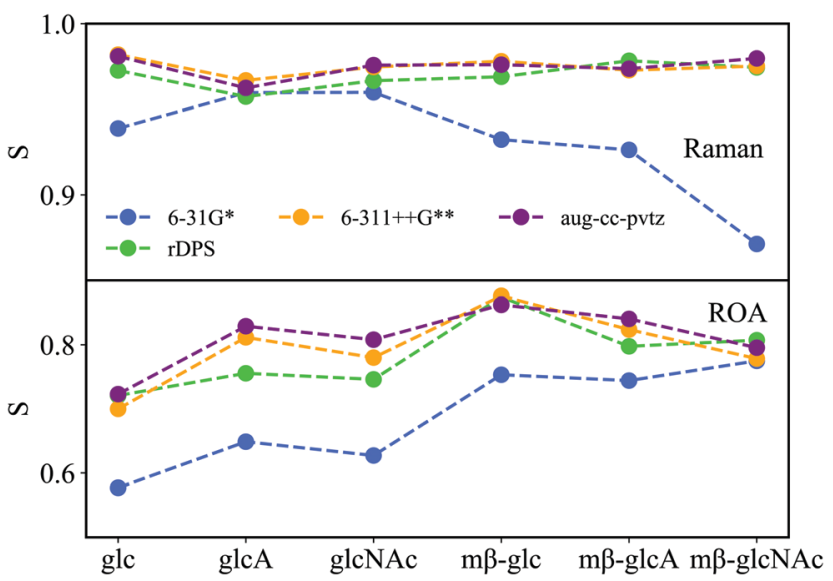

Fig. 8 Performance of the hybrid protocol when using different basis sets.
6-31G* and $\operatorname{rDPS}^{55}$ (see Table S1 in the ESI $\dagger$ ), the intermediate $6-311++G^{* *}$ and the expensive aug-cc-pvtz basis sets. Fig. 8 shows that the $6-31 \mathrm{G}^{*}$ basis set is not large enough to reproduce the spectral features, while $6-311++G^{* *}$ is sufficient and it performs almost the same as the much more expensive aug-ccpvtz. The frequently used rDPS basis set ${ }^{11,13,17-20}$ offers slightly worse performance compared to $6-311++\mathrm{G}^{* *}$ at the cost of the $\sim 6-31 \mathrm{G}^{*}$ basis set.

\subsection{Conformational sampling}

This work shows that a representative ensemble of structures is the most critical point for achieving accurate Raman and ROA spectra of saccharides. Not only do we need to properly sample the configurational space of the sugar, but we also need to map the sugar water environment reasonably well, to recover important spectral features. Two factors must be taken into account to properly describe the ensemble. The first one concerns structural nuances of the investigated sugar. Small differences in bond lengths, angles, and dihedral angles have a strong effect on the resulting spectrum. Fig. 9 (top panel) shows, for example, the superposition of spectra of different methyl $\beta$-D-glucuronide MD snapshots (black lines) obtained using the CPCM solvation approach (i.e., without explicit water molecules). The spectra of individual snapshots differ significantly from the average and experimental spectra, underscoring the effect of the sugar structural variability. Only sufficient averaging over a significant number of snapshots provides a computed spectrum (red line) that agrees reasonably with the experiment (blue line). Fig. S16 in the ESI† demonstrates that we need at least 30 snapshots to obtain a converged Raman spectrum and 80 snapshots to get a converged ROA spectrum of a monosaccharide.

Fig. 9 (middle panel) shows Raman and ROA spectra of methyl $\beta$-D-glucuronide using the hybrid approach (see Table 1).
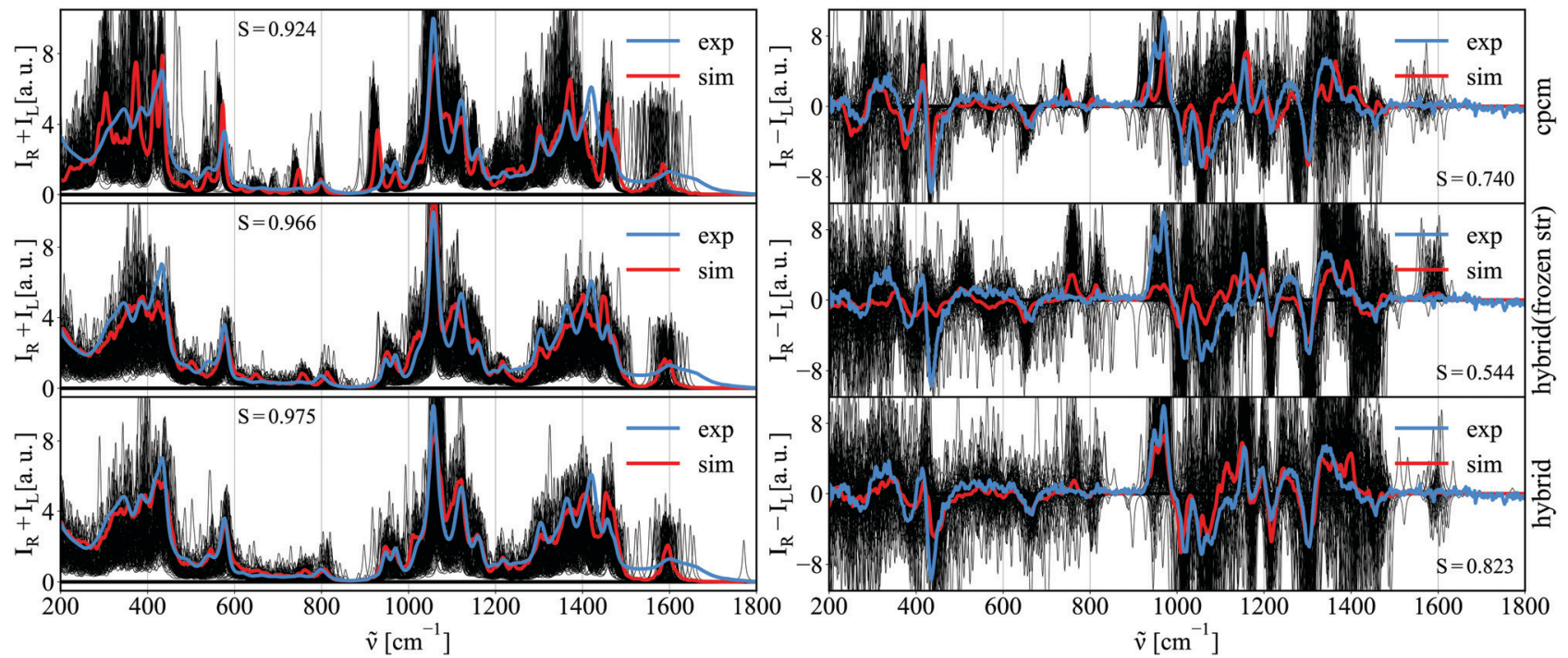

Fig. 9 Superposition of hundreds of Raman and ROA spectra of MD snapshots for methyl $\beta$-D-glucuronide (black), and the corresponding average spectrum (red) when using continuum (top) and hybrid (middle/bottom) solvation protocols on the same ensemble. In the middle panel, we show the effect of water molecules on the spectra using the hybrid (frozen carbohydrate structure) protocol. Experimental spectra are plotted in blue. 
In this case, the ensemble of MD structures was generated while the geometry of the monosaccharide was frozen in an arbitrarily chosen conformation $\left({ }^{4} C_{1}\right)$. It is striking that despite the fixed geometry of the saccharide the local water environment itself caused such a significant inhomogeneous broadening of the vibrational bands. Capturing both the sugar and water conformational freedom is thus essential for describing the key features of the spectra (see the bottom panel of Fig. 9).

\section{Discussion}

Contrary to other biomolecules the conformational behavior of saccharides is much less explored. Raman and Raman optical activity (ROA) spectroscopies appear as powerful tools for gaining valuable insight into the structure of saccharides.

In this paper, we performed computations of Raman and ROA spectra of monosaccharides in solution in a systematic way. We developed an optimized computational protocol to calculate the spectra for yielding quantitative or at least semiquantitative agreement with experiment. This protocol is applicable also to larger structures, which will be addressed in future studies. The spectra obtained for a test set of monosaccharides - methyl $\beta$-glucopyranoside, methyl $\beta$-D-glucuronide, methyl $\beta$ - $N$-acetyl-glucosaminide, D-glucose, D-glucuronic acid, and $N$-acetyl-D-glucosamine - agreed well with the experimental ones. Note that this set of monosaccharides comprises various sugar types, which suggests that the method can be applied to a large variety of sugar moieties.

An obvious but very important finding of this work is that there is not a single sugar structure that would fit the experimental spectrum, see Fig. 9. Instead, we show that a representative ensemble of structures is crucial to reproduce the Raman and ROA experimental spectra. Only averaging of individual spectra thus results in a good approximation to the experimental spectra. This behavior has been to some extent observed for other flexible polyhydroxylic molecules. ${ }^{54}$ Moreover, we show that both anomers have to be considered while aiming at simulation of reducing sugars. Failure to do so means essentially to partially simulate a different system from the one that is studied experimentally.

Although we achieved good accuracy in reproducing the spectra, a question arises about the source of the remaining errors and also about the inconsistencies for D-glucose and D-glucuronic acid. Therefore, full optimization of glucose together with using QM water molecules (Fig. S15 in the ESI $\dagger$ ) was performed. Overall, the data suggest that in the lower frequency region the description of water molecules mattered and the QM description was superior to MM. However, the calculations were not repeated for the rest of the considered molecules, since they are expensive. Another possible source of the errors is the incomplete conformational ensemble generated by molecular dynamics. We may be facing a force field problem, which goes beyond the scope of this work. Nevertheless, we could partially overcome this problem by using enhanced sampling techniques to find new configurations, followed by reconstruction of the spectra based on the experimental data.

Analyzing the main used protocols, in agreement with previous results, ${ }^{13}$ we show that continuum solvation models already capture some spectral features. When adding harmonic restraints, the spectra improved, e.g., methyl $\beta$-glucopyranoside. ${ }^{13}$ Overall, only using an implicit solvent model leads to apparent deficiencies in the calculated spectra, see e.g., the ROA spectra of D-glucuronic acid in Fig. S4 (top) in the ESI. $\dagger$ In order to obtain a spectrum that can be compared quantitatively with experimental data, we need at least to include the first solvation shell explicitly. This inclusion can be done by either using the MM solvation scheme, where hundreds of MM described water molecules surround the investigated saccharide, or utilizing the hybrid solvation model, which only includes the first coordination shell coupled with a continuum solvation model.

We further tested the hybrid protocol when limiting the number of QM optimization steps for each MD snapshot performed prior to calculation of the Raman/ROA properties. It turned out to be a critical variable. Around 10 optimizations steps proved to be optimal while further optimization only worsened both the Raman and ROA spectra. While 10 step optimization in the hybrid scheme yielded the best results, when using the MM scheme it performed rather poorly. We were able to recover most of the errors performing full optimization of the sugar while keeping the water molecules frozen (MM_w, i.e. the OPTSOLUTE approach used by the "Manchester group"13,17), however, at additional computational cost. Further comparison of hybrid_w and hybrid_fo showed that excessive optimization without any restraints only worsens the results. Overall, hybrid performs the best, while being computationally the most efficient.

In subsequent tests, we show that the impact of using different DFT functionals is small. From those tested B3LYP performed the best. On the other hand, the choice of basis set turned out to be important. Small basis set $6-31 G^{*}$ was not able to accurately reproduce the spectral features. The widely used rDPS basis set ${ }^{11,13,17-20}$ (see Table S1 in the ESI $\dagger$ ) provides significantly better results at a similar cost to the $6-31 \mathrm{G}^{*}$ basis set. Using the 6-311++G** basis set provides further improvement of the results at $\sim 2.5 \times$ the performance cost as compared to rDPS. Going even for larger and much more expensive aug-cc-pvtz provides only a little improvement. Therefore, we recommend $6-311++G^{* *}$ where the accuracy is crucial, while rDPS offers the best quality/cost ratio.

Unlike in most previous studies, we used an adaptable scaling function for vibrational frequencies $\phi\left(\tilde{\nu}^{\text {sim }}\right)$. As it ultimately allows for superposition of spectra it facilitated the visual comparison of the simulated spectra. Moreover, it increases the agreement with experimental data as with its use we obtain $\sim 97 \%$ overlap for Raman and $\sim 81 \%$ for ROA spectra.

Note that low frequencies at the B3LYP/6-311++G** level of theory practically do not need to be scaled, while high frequencies $\left(>1200 \mathrm{~cm}^{-1}\right)$ need to be scaled by a factor of $\sim 0.98$. This can be seen in Fig. S17 in the ESI, $\dagger$ where all optimized scaling 
Table 2 Comparison of the performance of the used protocols and their efficiency

\begin{tabular}{llll}
\hline Protocol & Rel. cpu time ${ }^{a}$ & $\left\langle S_{\text {Raman }}\right\rangle$ & $\left\langle S_{\text {ROA }}\right\rangle$ \\
\hline CPCM & 1 & 0.941 & 0.698 \\
MM & 3.7 & 0.952 & 0.761 \\
MM_w & 7.5 & 0.966 & 0.778 \\
hybrid & 1.7 & 0.974 & 0.805 \\
hybrid_w & 3 & 0.968 & 0.773 \\
hybrid_fo & 14 & 0.954 & 0.735 \\
${ }^{a}$ With respect to CPCM. & & \\
\end{tabular}

functions are shown. Optimizing the scaling parameters for each simulation is certainly desirable and for each of the protocols a unique average scaling function exists. For the hybrid protocol we recommend $\phi\left(\tilde{\nu}^{\operatorname{sim}}, a, b, c, d\right)=\phi\left(\tilde{\nu}^{\text {sim }}, 0.982,1.00,15,1210\right)$, while for the rDPS protocol (see Table S1 in the ESI $\dagger$ ) we recommend $\phi\left(\tilde{\nu}^{\operatorname{sim}}, a, b, c, d\right)=\phi\left(\tilde{\nu}^{\operatorname{sim}}, 0.957,0.983,40,1437\right)$.

A comparison of the performance in the relative cpu times with respect to CPCM can be found in Table 2, where it is shown that the hybrid approach provides the best quality/cost ratio.

Lastly, we show with glucose that using QM water molecules rather than MM makes a small difference at high computational cost (relative cpu times with respect to hybrid are hybrid: hybrid_QM : hybrid_QM_fo = 1:25:70; Raman overlap integral: $0.983: 0.978: 0.980$; ROA overlap integral: $0.756: 0.635: 0.720$ ).

\section{Conclusions}

We have developed and tested a computational protocol (hybrid, see Table 1) designed to accurately and efficiently simulate Raman and ROA spectra of saccharides. This hybrid protocol is based on MD simulations. A sufficient number of snapshots $(\sim 100)$ is extracted, and only water molecules closer than $3 \AA$ to the solute are kept. The long range solvation effects are modeled by the CPCM continuum model. The snapshots are then optimized using a 10 step optimization. The QM calculations are performed at the B3LYP/6-311++G** level of theory while explicit water molecules are treated at the MM level. This approach provides good agreement with experimental data, with residual inconsistencies for Raman and ROA intensities, being accurate enough to determine the conformational preferences of the sugars. Special attention is paid to making the protocol computationally efficient so that it can also be used for larger oligosaccharides. Simulations of the Raman and ROA intensities thus make it possible to extract detailed information about the structure of saccharides in water solutions.

\section{Conflicts of interest}

There are no conflicts to declare.

\section{Acknowledgements}

Access to computing and storage facilities owned by parties and projects contributing to the National Grid Infrastructure
MetaCentrum provided under the programme "Projects of Large Research, Development, and Innovations Infrastructures" (CESNET LM2015042), is greatly appreciated. HMS, JK, and VP acknowledge support from the Czech Science Foundation (19-19561S). PB acknowledges support from the Ministry of Education (LTC17012). PJ acknowledges support from the European Regional Development Fund OP RDE (project ChemBioDrug no. CZ.02.1.01/0.0/0.0/16_019/0000729).

\section{References}

1 J. M. Tarbell and M. Y. Pahakis, J. Intern. Med., 2006, 259, 339-350.

2 J. M. Tarbell and L. M. Cancel, J. Intern. Med., 2016, 280, 97-113.

3 R. R. Kapaev, K. S. Egorova and P. V. Toukach, J. Chem. Inf. Model., 2014, 54, 2594-2611.

4 O. Dahlman, A. Jacobs, A. Liljenberg and A. I. Olsson, J. Chromatogr. A, 2000, 891, 157-174.

5 K. Matsuo, H. Namatame, M. Taniguchi and K. Gekko, J. Phys. Chem. A, 2012, 116, 9996-10003.

6 K. Matsuo, Biomed. Spectrosc. Imaging, 2017, 6, 111-121.

7 L. D. Barron, M. P. Bogaard and A. D. Buckingham, J. Am. Chem. Soc., 1973, 95, 603-605.

8 N. R. Yaffe, A. Almond and E. W. Blanch, J. Am. Chem. Soc., 2010, 132, 10654-10655.

9 T. R. Rudd, R. Hussain, G. Siligardi and E. A. Yates, Chem. Commun., 2010, 46, 4124-4126.

10 V. Profant, C. Johannessen, E. W. Blanch, P. Bouř and V. Baumruk, Phys. Chem. Chem. Phys., 2019, 21, 7367-7377.

11 S. T. Mutter and E. W. Blanch, Polysaccharides, Springer International Publishing, 2015, pp. 1181-1218.

12 A. Melcrová, J. Kessler, P. Bouř and J. Kaminský, Phys. Chem. Chem. Phys., 2016, 18, 2130-2142.

13 J. R. Cheeseman, M. S. Shaik, P. L. A. Popelier and E. W. Blanch, J. Am. Chem. Soc., 2011, 133, 4991-4997.

14 T. Giovannini, M. Olszówka, F. Egidi, J. R. Cheeseman, G. Scalmani and C. Cappelli, J. Chem. Theory Comput., 2017, 13, 4421-4435.

15 S. Luber and M. Reiher, J. Phys. Chem. A, 2009, 113, 8268-8277.

16 N. A. Macleod, C. Johannessen, L. Hecht, L. D. Barron and J. P. Simons, Int. J. Mass Spectrom., 2006, 253, 193-200.

17 R. Pendrill, S. T. Mutter, C. Mensch, L. D. Barron, E. W. Blanch, P. L. A. Popelier, G. Widmalm and C. Johannessen, ChemPhysChem, 2019, 20, 695-705.

18 F. Zielinski, S. T. Mutter, C. Johannessen, E. W. Blanch and P. L. A. Popelier, Phys. Chem. Chem. Phys., 2015, 17, 21799-21809.

19 S. T. Mutter, F. Zielinski, C. Johannessen, P. L. A. Popelier and E. W. Blanch, J. Phys. Chem. A, 2016, 120, 1908-1916.

20 S. T. Mutter, F. Zielinski, J. R. Cheeseman, C. Johannessen, P. L. A. Popelier and E. W. Blanch, Phys. Chem. Chem. Phys., 2015, 17, 6016-6027. 
21 A. Rüther, A. Forget, A. Roy, C. Carballo, F. Mießmer, R. K. Dukor, L. A. Nafie, C. Johannessen, V. P. Shastri and S. Lüdeke, Angew. Chem., Int. Ed., 2017, 56, 4603-4607.

22 Y. Zhu, J. Zajicek and A. S. Serianni, J. Org. Chem., 2001, 66, 6244-6251.

23 V. Profant, M. Pazderková, T. Pazderka, P. Maloň and V. Baumruk, J. Raman Spectrosc., 2014, 45, 603-609.

24 M. J. Abraham, T. Murtola, R. Schulz, S. Páll, J. C. Smith, B. Hess and E. Lindahl, SoftwareX, 2015, 1-2, 19-25.

25 D. E. Smith and L. X. Dang, J. Chem. Phys., 1994, 100, 3757-3766.

26 K. N. Kirschner, A. B. Yongye, S. M. Tschampel, J. GonzálezOuteiriño, C. R. Daniels, B. L. Foley and R. J. Woods, J. Comput. Chem., 2008, 29, 622-655.

27 S. Izadi and A. V. Onufriev, J. Chem. Phys., 2016, 145, 074501.

28 T. Darden, D. York and L. Pedersen, J. Chem. Phys., 1993, 98, 10089-10092.

29 M. R. Shirts, D. L. Mobley, J. D. Chodera and V. S. Pande, J. Phys. Chem. B, 2007, 111, 13052-13063.

30 B. Hess, H. Bekker, H. J. C. Berendsen and J. G. E. M. Fraaije, J. Comput. Chem., 1997, 18, 1463-1472.

31 S. Nosé, J. Chem. Phys., 1984, 81, 511-519.

32 W. G. Hoover, Phys. Rev. A: At., Mol., Opt. Phys., 1985, 31, 1695-1697.

33 M. Parrinello and A. Rahman, J. Appl. Phys., 1981, 52, 7182-7190.

34 M. J. Frisch, G. W. Trucks, H. B. Schlegel, G. E. Scuseria, M. A. Robb, J. R. Cheeseman, G. Scalmani, V. Barone, G. A. Petersson, H. Nakatsuji, X. Li, M. Caricato, A. V. Marenich, J. Bloino, B. G. Janesko, R. Gomperts, B. Mennucci, H. P. Hratchian, J. V. Ortiz, A. F. Izmaylov, J. L. Sonnenberg, D. Williams-Young, F. Ding, F. Lipparini, F. Egidi, J. Goings, B. Peng, A. Petrone, T. Henderson, D. Ranasinghe, V. G. Zakrzewski, J. Gao, N. Rega, G. Zheng, W. Liang, M. Hada, M. Ehara, K. Toyota, R. Fukuda, J. Hasegawa, M. Ishida, T. Nakajima, Y. Honda, O. Kitao, H. Nakai, T. Vreven, K. Throssell, J. A. Montgomery Jr., J. E. Peralta, F. Ogliaro, M. J. Bearpark, J. J. Heyd, E. N. Brothers, K. N. Kudin, V. N. Staroverov, T. A. Keith, R. Kobayashi, J. Normand, K. Raghavachari, A. P. Rendell, J. C. Burant, S. S. Iyengar, J. Tomasi, M. Cossi, J. M. Millam, M. Klene, C. Adamo, R. Cammi, J. W. Ochterski, R. L. Martin, K. Morokuma, O. Farkas, J. B. Foresman and D. J. Fox, Gaussian16 Revision B.01, 2016.
35 L. Nafie, Vibrational optical activity: Principles and applications, Wiley, Chichester, 2011.

36 K. Ruud, T. Helgaker and P. Bouř, J. Phys. Chem. A, 2002, 106, 7448-7455.

37 S. Dapprich, I. Komáromi, K. S. Byun, K. Morokuma and M. J. Frisch, THEOCHEM, 1999, 461-462, 1-21.

38 M. Orozco, I. Marchán, I. Soteras, T. Vreven, K. Morokuma, K. V. Mikkelsen, A. Milani, M. Tommasini, M. D. Zoppo, C. Castiglioni, M. A. Aguilar, M. L. Sánchez, M. E. Martín, I. Fdez Galván and H. Sato, in Continuum solvation models in chemical physics, ed. B. Mennucci and R. Cammi, John Wiley \& Sons, Ltd, Chichester, UK, 2007, pp. 499-605.

39 W. L. Jorgensen, J. Chandrasekhar, J. D. Madura, R. W. Impey and M. L. Klein, J. Chem. Phys., 1983, 79, 926-935.

40 G. E. Scuseria, C. A. Jiménez-Hoyos, T. M. Henderson, K. Samanta and J. K. Ellis, J. Chem. Phys., 2011, 135, 124108. 41 P. L. Polavarapu, J. Phys. Chem., 1990, 94, 8106-8112.

42 M. K. Kesharwani, B. Brauer and J. M. L. Martin, J. Phys. Chem. A, 2015, 119, 1701-1714.

43 M. L. Laury, M. J. Carlson and A. K. Wilson, J. Comput. Chem., 2012, 33, 2380-2387.

44 P. Sinha, S. E. Boesch, C. Gu, R. A. Wheeler and A. K. Wilson, J. Phys. Chem. A, 2004, 108, 9213-9217.

45 P. M. Jeffrey, D. Moran and L. Radom, J. Phys. Chem. B, 2007, 111, 11683-11700.

46 W. A. Bubb, Concepts Magn. Reson., Part A, 2003, 19, 1-19.

47 F. Franks, P. J. Lillford and G. Robinson, J. Chem. Soc., Faraday Trans. 1, 1989, 85, 2417.

48 T. Bezabeh, O. B. Ijare, N. Albiin, U. Arnelo, B. Lindberg and I. C. P. Smith, Magn. Reson. Mater. Phys., Biol. Med., 2009, 22, 267-275.

49 B. Moreau, G. Lognay, C. Blecker, J. Destain, P. Gerbaux, F. Chéry, P. Rollin, M. Paquot and M. Marlier, Biotechnol. Agron. Soc. Environ., 2007, 11, 9-17.

50 F. C. Liu, C. R. Su, T. Y. Wu, S. G. Su, H. L. Yang, J. H. Y. Lin and T. S. Wu, Int. J. Mol. Sci., 2011, 12, 5828-5843.

51 P. Bouř and T. A. Keiderling, J. Chem. Phys., 2002, 117, 4126-4132.

52 S. Yamamoto, J. Kaminský and P. Bouř, Anal. Chem., 2012, 84, 2440-2451.

53 V. Parchaňský, J. Kapitán, J. Kaminský, J. Šebestík and P. Bouř, J. Phys. Chem. Lett., 2013, 4, 2763-2768.

54 J. Kaminský, J. Kapitán, V. Baumruk, L. Bednárová and P. Bouř, J. Phys. Chem. A, 2009, 113, 3594-3601.

55 G. Zuber and W. Hug, J. Phys. Chem. A, 2004, 108, 2108-2118. 